\title{
A Sustained-Release Membrane of Thiazolidinedione-8: Effect on Formation of a Candida/Bacteria Mixed Biofilm on Hydroxyapatite in a Continuous Flow Model
}

\author{
Mark Feldman, ${ }^{1}$ Julia Shenderovich, ${ }^{1,2}$ Eran Lavy, ${ }^{3}$ \\ Michael Friedman, ${ }^{2}$ and Doron Steinberg ${ }^{1}$ \\ ${ }^{1}$ Biofilm Research Laboratory, Institute of Dental Sciences, Faculty of Dental Medicine, \\ The Hebrew University of Jerusalem, Jerusalem, Israel \\ ${ }^{2}$ The Institute for Drug Research, School of Pharmacy, The Hebrew University of Jerusalem, Jerusalem, Israel \\ ${ }^{3}$ Koret School of Veterinary Medicine, Robert H. Smith Faculty of Agriculture, Food and Environment, \\ The Hebrew University of Jerusalem, Rehovot, Israel
}

Correspondence should be addressed to Doron Steinberg; dorons@ekmd.huji.ac.il

Received 27 June 2017; Revised 9 August 2017; Accepted 30 August 2017; Published 10 October 2017

Academic Editor: Viness Pillay

Copyright (C) 2017 Mark Feldman et al. This is an open access article distributed under the Creative Commons Attribution License, which permits unrestricted use, distribution, and reproduction in any medium, provided the original work is properly cited.

\begin{abstract}
Thiazolidinediones (TZDs) have been found to act as effective quorum sensing quenchers, capable of preventing biofilm formation. Our previous studies demonstrated a profound antibiofilm effect of the TZD derivative thiazolidinedione-8 (S-8), either in solution or incorporated into a sustained-release membrane (SRM-S-8) under batch conditions. In the present study, we used a constant depth film fermenter model in order to investigate the impact of SRM-S-8 on mixed C. albicans-S. mutans biofilm development, under flow conditions. We found that essential parameters of cospecies biofilm maintenance and maturation, such as metabolic activity, biofilm thickness, roughness, extracellular polysaccharides production, and morphology of both pathogens, were altered by SRM-S- 8 in the flow system. We propose that prolonged and sustained release of S- 8 in a flow-through system allows better penetration of the active agent to deeper layers of the mixed biofilm, thereby increasing its activity against both pathogens. In conclusion, the use of a locally applied sustained-release drug delivery system of S-8 can affect the dental polymicrobial biofilm, resulting in clinical improvements and a better patient compliance.
\end{abstract}

\section{Introduction}

The oral cavity is colonized by a plethora of microbial species, most of which can form biofilms that adhere to the dental oral surfaces.

The fungus Candida albicans is a human pathogen that is also found in the oral cavity. It is a dimorphic fungus that grows as both yeast and filamentous cells, and its local propagation leads to a fungal infection known as candidiasis. The oral clinical signs and symptoms of this infection include white patches on the soft tissue of the mouth, soreness, and problems with swallowing. Candidal biofilm formation is associated with extracellular polysaccharides (EPS) production and a morphologic switch to a pathogenic hyphal form [1].
Streptococcus mutans has long been recognized as one of the major causes of dental caries [2] due to its acidogenic and aciduric properties as well as its high biofilm formation efficiency. S. mutans biofilms progress when bacteria utilize dietary sugars and proliferate on tooth surface, forming microcolonies that are firmly imbedded within EPS [3]. EPS are synthesized by the bacterial extracellular enzymes glucosyltransferases (GTFs) and fructosyltransferases (FTFs) [4]. Moreover, EPS type glucans, synthesized by extracellular GTF, provide binding sites for bacterial colonization, thus promoting further development of the biofilms [5].

Cospecies communities of C. albicans and S. mutans are often found in the oral cavity. C. albicans has been found in close association with oral streptococci in denture- and mucosal-related diseases [6]. The coexistence of these species 
was demonstrated in early childhood caries [7] and also in deep carious lesions [8]. Streptococcus gordonii and Streptococcus oralis form dual species biofilms with C. albicans on salivary glycoprotein-coated surfaces [9] or on epithelium [10]. Several species of oral streptococci promote growth, hyphae formation [11, 12], and virulence of C. albicans [13, 14]. Collaboration occurring in dual species biofilms of $S$. mutans and C. albicans leads to increased biofilm mass and cell densities $[7,15]$, thereby enhancing biofilm pathogenicity. It was shown that farnesol, a fungal quorum sensing (QS) signal, induces $S$. mutans biofilm cell growth, microcolony development, and GTF expression and activity [16]. The GTF$B$ enzyme exhibits high binding affinity to C. albicans [17, 18].

Oral infections such as candidiasis, caries, periodontal diseases, and root canal infections are of chronic nature; therefore prolonged therapy is required. However, most dental drugs are rapidly removed from the oral cavity; therefore, there is a need to develop sustained-release delivery systems which can prolong the time of residence of the drug at the active site [19]. Local sustained-release delivery systems increase the efficacy and the safety of the administered drug [20]. More so, due to the prolonged substantiality of the drug released from the sustained-release delivery system its effect also on the biofilm is more profound [21].

Sustained-release delivery systems, including varnishes and membranes intended for the oral cavity were able to maintain therapeutic concentrations of the active agent in the oral cavity for prolonged periods, along with negligible blood concentrations [22]. Several studies have demonstrated the in vivo effectiveness of antimicrobial or antifungal agents incorporated into sustained-release delivery systems, against S. mutans [23] or C. albicans [22, 24].

Thiazolidinediones (TZDs) have been documented as effective antibiofilm, quorum sensing quenchers [1,25-28], which have the capacity to prevent biofilm formation in vitro. We found a profound antibiofilm effect of the TZD derivative, thiazolidinedione- 8 (S-8), in solution $[1,29]$. In order to enhance its biological activity, the TZD was incorporated into a sustained-release membrane (SRM) [28]. It was demonstrated that a SRM, containing the TZD derivative S-8 (SRM-S-8), inhibits fungal biofilm formation in a timedependent manner, due to the prolonged release kinetics of S-8. Moreover, the TZD released from the membrane induced a significant decrease in the biofilm formed on the SRM-surrounding area. In addition, virulent parameters of matured biofilm such as attachment force, thickness, exopolysaccharide production, and morphogenesis of fungal cells were dramatically altered by SRM-S-8 [28]. It was also found that in its soluble form this agent alters the symbiotic relationship in the C. albicans-S. mutans dual species biofilm in a batch system [30].

For further investigating biofilm formation in the oral cavity, model systems are needed which provide constant flow conditions in combination with low shear forces. The constant depth film fermenter (CDFF) is widely used for the research of dental biofilm development [31-35]. The CDFF represents a flow system allowing for controlling the biofilm depth, flow of nutrients, and evacuation of metabolites.
In the present study we explored the antibiofilm effect of S-8, incorporated into sustained-release varnish membrane (SRM-S-8), on biofilm formation, using CDFF as a flow system and hydroxyapatite tablets (HA) as the surface.

\section{Materials and Methods}

2.1. Coating of the Hydroxyapatite Tablets. The SRM was prepared by dissolving polyethylene glycol (PEG-400, SigmaAldrich, Belgium), ammonio methacrylate copolymer type A NF (Eudragit ${ }^{\circledR}$ RL, Evonik Röhm GmbH, Germany), and S-8 (Daren Labs, Israel) in absolute ethanol (J. T. Baker, USA) until a homogenous solution was obtained [27]. The concentration of S-8 in the SRM was $20 \%$ of the dry weight. In order to determine the direct effect of SRM-S-8, one side of $9.5 \mathrm{~mm}$ diameter hydroxyapatite (HA) tablets (Clarkson Chromatography Products Inc., South Williamsport, PA, USA) was coated with the SRM preparation. The coated HA tablets were left to dry overnight at room temperature (RT). The tablets were weighed before and after coating, and the amount of S-8 was calculated as its weight percentage of the SRM-S-8.

2.2. Dissolution Studies. In vitro dissolution studies were performed at $37^{\circ} \mathrm{C}$ in a flow-through system using a syringe pump. Briefly, each HA tablet was inserted into a flowthrough chamber (prepared in-house) of an approximate volume of $1.5 \mathrm{ml}$, attached to a $30 \mathrm{ml}$ syringe filled with $1 \%(\mathrm{w} / \mathrm{v})$ of sodium lauryl sulfate (Sigma-Aldrich) in water as the release medium. The syringes were then connected to a syringe pump (Harvard Apparatus 22, USA) that forced the liquid through the chamber and into a collecting tube at a rate of $1.25 \mathrm{ml} / \mathrm{h}$. After $24 \mathrm{~h}$, the syringes were refilled with $30 \mathrm{ml}$ of the release medium, and the flow-through process continued for another 24 hours. The collecting tubes were replaced at designated time points, and S-8 concentrations in the samples were determined by HPLC. At the end of the experiment, each HA tablet was immersed in absolute ethanol to dissolve the remaining $\mathrm{S}-8$, in order to determine the residual concentration that had not been released.

2.3. S-8 Analysis. The amount of S-8 released from the SRM-S- 8 coated HA was determined by HPLC, using HP 1050 HPLC equipped with a UV detector. Separation was performed on a Hypersil Gold C-18 column $(250 \times$ $4.6 \mathrm{~mm} 5 \mu \mathrm{m}$, Thermo Scientific, USA). The mobile phase was acetonitrile: water $(70: 30 \% \mathrm{v} / \mathrm{v})$ and the flow rate was $1 \mathrm{ml} / \mathrm{min}$. S-8 was detected at $280 \mathrm{~nm}$. The calibration curve was linear at $1.5-100 \mu \mathrm{g} / \mathrm{ml}$ range [27].

2.4. CDFF Flow System. The controlled release film fermenter (CDFF) consists of a glass vessel which houses a stainless steel rotating disc, containing 15 PTFE plugs (18.0 mm diameter) $[31,32]$. The HA tablets were placed onto the plugs. The surface level of the plugs can be controlled to ensure a constant depth of the biofilm formed on top of the HA tablets. As the turntable rotates, a PTFE scraper bar moves on the surface of the disc. The PTFE bar allows the incoming 


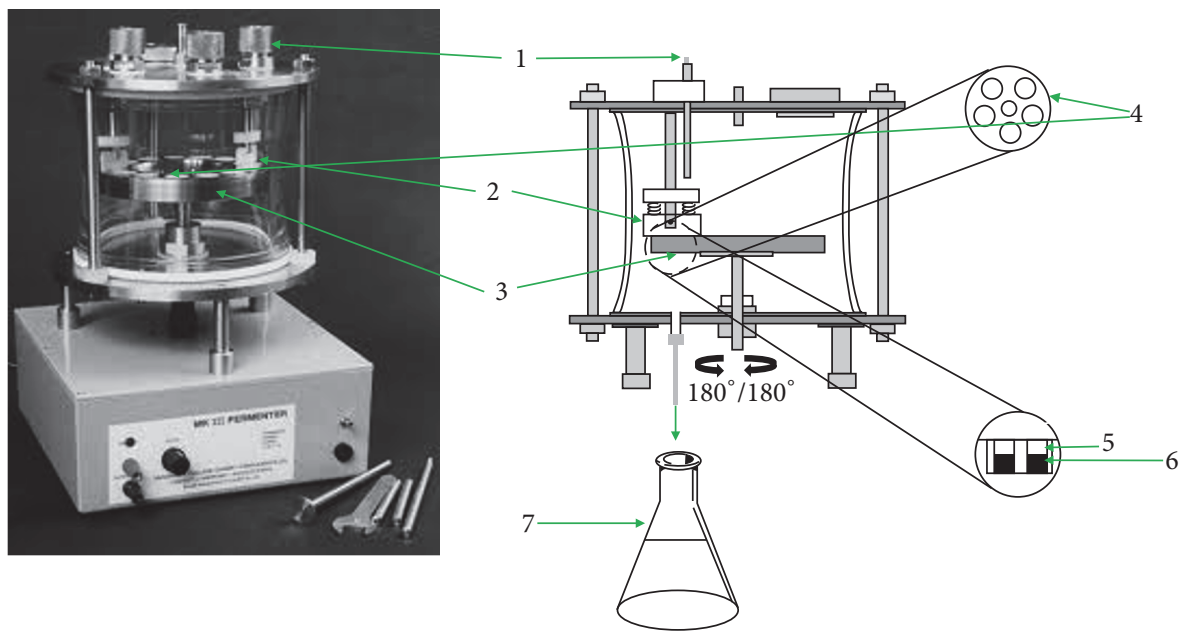

FIGURE 1: CDFF flow system (Greenman et al., 2013; with few modifications made by authors). (1) Sample port $\times 3$. (2) PTFE scraper bar $\times 2$. (3) Turntable. (4) PTFE plug $\times$ 15. (5) Formed biofilm. (6) HA tablet-SRM (control/S-8). (7) Waste flask.

liquid to reach the biofilm and maintains the biofilms, once formed, at a constant predetermined depth (Figure 1). Two 2 L flasks, containing BHI medium, were connected through a peristaltic pump to the incoming sample ports of the glass vessel, while another $5 \mathrm{~L}$ flask was connected to the waste port (Figure 1).

The CDFF system was autoclaved at $121^{\circ} \mathrm{C}$ for $60 \mathrm{~min}$ before use. After autoclaving, SRM and SRM-S-8 coated HA tablets were aseptically placed on top of the PTFE plugs and adjusted to a depth of $100 \mu \mathrm{m}$. Next, $1 \%$ of sucrose was added aseptically to the flask containing BHI which served as a supplemented medium, while another flask with BHI was inoculated with the microbes, as described below.

\subsection{Dual Species Biofilm Development in CDFF. Precultures} of S. mutans UA159 and C. albicans SC5314 were inoculated from several isolated colonies, grown separately for $18 \mathrm{~h}$, in $5 \% \mathrm{CO}_{2}$ at $37^{\circ} \mathrm{C}$, and harvested by centrifugation $(3136 \mathrm{~g}$, $\left.10 \mathrm{~min}, 4^{\circ} \mathrm{C}\right)$. Next, both microbial cultures were diluted to $\mathrm{OD}_{595}=0.1 \mathrm{in} \mathrm{BHI}$ medium and incubated overnight, in 5\% $\mathrm{CO}_{2}$ at $37^{\circ} \mathrm{C}$ in $2 \mathrm{~L} \mathrm{BHI}$ containing flask. After incubation the flask was connected to the CDFF as described below.

The assay was performed similarly as described previously [33]. Briefly, biofilms were allowed to develop in CDFF on SRM/SRM-S- 8 coated HA tablets for $48 \mathrm{~h}$, in a $5 \% \mathrm{CO}_{2}$ atmosphere, at $37^{\circ} \mathrm{C}$. The mixed inoculum and supplemented medium were pumped into the CDFF just in front of the PTFE scraper blade at a rate of $40 \mathrm{ml} / \mathrm{h}$. The turntable rotated at a speed of $3 \mathrm{rpm}$ and the fermenter was supplied with air, which was allowed to diffuse through sterile filters. The CDFF was operated in reciprocal mode ( $180^{\circ}$ oscillation) (Figure 1). In order to prevent any contact between treated and untreated biofilms, SRM coated HA tablets were used as a placebo control on one side of the rotating disc, while SRM-S- 8 coated HA tablets were inserted on the opposite side of the disc. Therefore, the liquids removed by the scraper blade from control and treated samples did not interact. After biofilm formation, all HA tablets were removed aseptically from the CDFF, washed 3 times in sterile PBS, and processed for further analysis as described below.

2.6. Microbial Metabolic Activity of the Formed Biofilms. The assay measuring metabolic activity was performed as described previously [30], with several modifications. Briefly, the mixed biofilms of $C$. albicans and $S$. mutans formed on SRM/SRM-S-8 coated HA tablets in the CDFF were washed with sterile PBS. Next the biofilm was overlaid with $100 \mathrm{mM}$ of 3-(4,5-dimethyl-2-thiazolyl)-2,5-diphenyl$2 \mathrm{H}$-tetrazolium bromide (MTT) and incubated for $2 \mathrm{~h}$ at $37^{\circ} \mathrm{C}$. Under these conditions, the lightly yellowish MTT is reduced to a blue tetrazolium salt accumulated within the metabolic active biofilms. The dye on the HA tablets was dissolved in DMSO and the absorbance value was measured at $570 \mathrm{~nm}$. The accumulation of tetrazolium salt by the reduction of MTT is proportional to the number of metabolically active cells growing in biofilm. Three independent experiments were performed.

2.7. Morphology of Mixed Biofilm. The assay was performed similarly, as described previously [30]. After washing, the HA tablets were immersed in $4 \%$ formaldehyde for $1 \mathrm{~h}$ at RT. The bacteria and fungi in the mixed biofilms formed on SRM/SRM-S-8 coated HA tablets were then visualized using an analytical Quanta 200 Environmental High Resolution Scanning Electron Microscope (EHRSEM) (FEI, Eindhoven, The Netherlands) at 2,000x magnification. At least three random fields were observed and analyzed. Three independent experiments were performed.

2.8. Confocal Laser Scanning Microscopy (CLSM) of Mixed Biofilms. The assay was performed similarly, as described previously [7,30]. Mixed inoculum was prepared as described above, but instead of C. albicans SC5314 wild type we used 
strain C. albicans SC5314 carrying the GFP reporter gene (C. albicans, GFP) [36], (green color) kindly provided by J. Berman (Tel Aviv University, Israel). In order to label S. mutans EPS, $1 \mathrm{mM}$ of Alexa Fluor 555-labeled dextran conjugate (red color) (10,000 MW, Molecular Probes Inc., Eugene, OR, USA) was added to the medium prior to biofilm formation, as described previously [30, 37]. Fortyeight-hour biofilms, developed on SRM/SRM-S- 8 coated HA tablets, were washed with PBS and incubated for $45 \mathrm{~min}$ with concanavalin A-Alexa Fluor 647 conjugate (ConA; $25 \mathrm{mg} / \mathrm{ml}$ ) (Invitrogen, Carlsbad, CA, USA). Con A (excitation wavelength $650 \mathrm{~nm}$ and emission at $668 \mathrm{~nm}$ ) (yellow color) selectively binds to the glucose and mannose residues of fungal cell wall exopolysaccharides (EPS) [30, 38]. Finally, streptococcal cells in cospecies biofilms were labeled by an immunofluorescence method developed in our laboratory and published in Abcam Review (2016, July). Briefly, after fungal EPS staining, biofilms were washed with PBS and fixed in $4 \%$ formaldehyde for $1 \mathrm{~h}$ at RT. Next, they were incubated for $1 \mathrm{~h}$ in PBS containing $1 \%$ bovine serum albumin (BSA) and then with rabbit antiStreptococcus mutans polyclonal antibody (1:500; Abcam, Cambridge, UK) in PBS-1\% BSA ( $1.5 \mathrm{~h}$ ) followed by Alexa Fluor 405-conjugated goat anti-rabbit IgG H\&L antibody (blue color) (1:500; Abcam) in PBS-1\% BSA (1h). Stained EPS and microorganisms were observed with a Zeiss LSM 510 CLS microscope (Carl Zeiss, Oberkochen, Germany). Three-dimensional images of the microbes and EPS distribution within biofilms were constructed using Zen2009 software (Carl Zeiss). At least three random fields were observed and analyzed, and three independent experiments were performed. The amount of each microbial species as well as individual EPS production by $S$. mutans and C. albicans in each sample was calculated as a colorappropriated fluorescence intensity, using ImageJ v3.91 software (http://rsb.info.nih.gov/ij). The data are presented as amount of fungal and bacterial cells as well as individual EPS production by C. albicans and S. mutans cells in each layer of the biofilm $(5 \mu \mathrm{m})$. The percentage of total EPS production and total biomass in mixed biofilms formed on SRM-S-8 coated HA tablets was calculated as area under the curve (AUC) and compared to control SRM coated HA tablets $(100 \%)$.

2.9. Surface Roughness of Formed Biofilms. Surface roughness of formed biofilms was evaluated using a Veeco Dektak 150 profilometer (DektakXT, Bruker, Bruker Nano Surfaces Division, Tucson, AZ, USA) [39]. Surface roughness was determined as average roughness (Sa) and root mean square roughness $(\mathrm{Sq})$. The $\mathrm{Sa}$ and $\mathrm{Sq}$ parameters represent an overall measurement of the biofilm surface topography. Sa expresses an absolute value of the differences in height of each point compared to the arithmetical mean of the biofilm surface. Sq represents the root mean square value of height values within the biofilm area. The profilometer was operated as follows: a $2 \mu \mathrm{m}$ stylus was moved in contact with the tested specimen during $60 \mathrm{sec}$, with a $1 \mathrm{mg}$ force, and on $1.078 \mathrm{~mm}$ from the center of the slab. Data were acquired and analyzed with the Vision $64^{\circledR} 5.40$ software (Bruker Corp., USA).

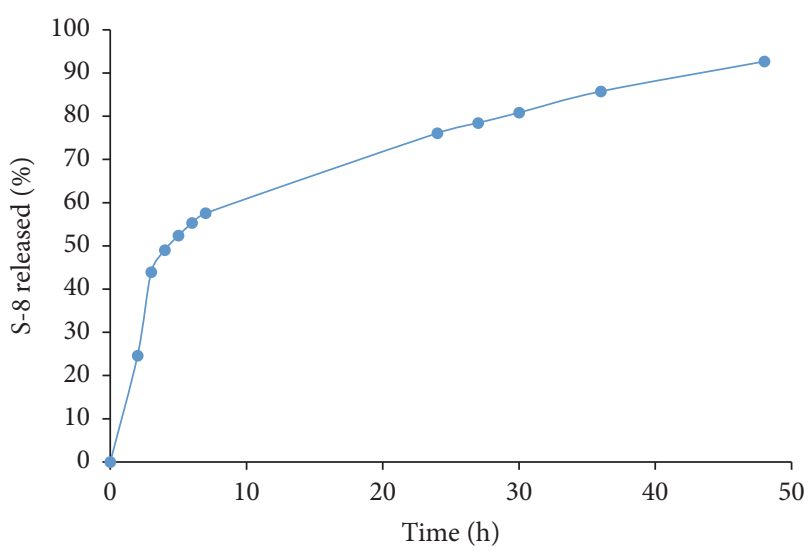

FIGURE 2: S-8 release kinetics from SRM coated hydroxyapatite tablets in a flow-through system. Release of S-8 is expressed as percentage of total S- 8 dose, where 100 percent represents the amount of S8 released in 48 hours plus the amount of residual unreleased S- 8 . Three independent experiments were performed.

2.10. Statistical Analysis. Means of three independent experiments were calculated. The statistical analysis was performed using Student's $t$-test with a significance level of $P<0.01$ as compared to controls.

\section{Results}

3.1. Kinetics of S-8 Release from the SRM-S-8-Coated HA in a Flow-Through System. The results shown in Figure 2 demonstrate the release of S- 8 from the SRM coated HA tablets over a period of $48 \mathrm{~h}$. A gradual release of the drug was detected during the initial $3 \mathrm{~h}$. A total of $44 \%$ of the S- 8 was released from the SRM within the initial $3 \mathrm{~h}$. However, after this time period, the release rate of S- 8 decreased, reaching $72 \%$ after $24 \mathrm{~h}$. A total of $92 \%$ of the S- 8 incorporated into the SRM coated HA tablets was released over the $48 \mathrm{~h}$.

3.2. Metabolic Activity of Mixed Biofilm Cells. The MTT assay was conducted to determine the effect of SRM-S-8 on biofilm metabolic activity. A significant decrease in metabolic activity of biofilms formed on SRM-S-8 coated HA tablets was observed (Figure 3(b)) compared to control SRM biofilms (Figure 3(a)). The amount of metabolically active microbial cells was reduced by $65 \%(P<0.01)$ in biofilms treated with SRM-S-8 $\left(\mathrm{OD}_{595}\right.$ of $1: 10$ diluted sample $=0.16+/-0.078^{*}$ $(35 \%))$ as compared to control $\left(\mathrm{OD}_{595}\right.$ of $1: 10$ diluted sample $=0.45+/-0.68(100 \%))$.

3.3. Morphology of Mixed Biofilms. SEM images showed that S-8 incorporated into SRM had a profound influence on the morphology and structure of mixed biofilms developed on the HA tablets. As shown in Figure 4, untreated control biofilm contained long matured filaments of C. albicans forming well-developed mycelium, while the streptococcal cells appeared in a form of numerous clusters attached to fungal hyphae cells (Figure 4(a)). In contrast, biofilms formed on HA tablets coated with SRM-S-8 consisted of candidal yeast form only, while bacteria were mostly in a single chain 


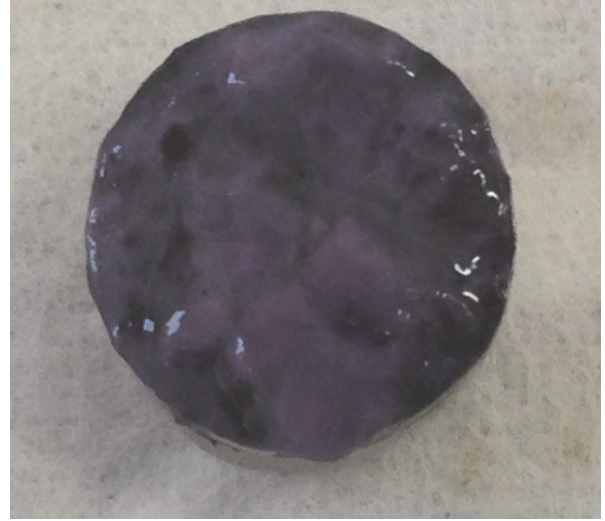

(a)

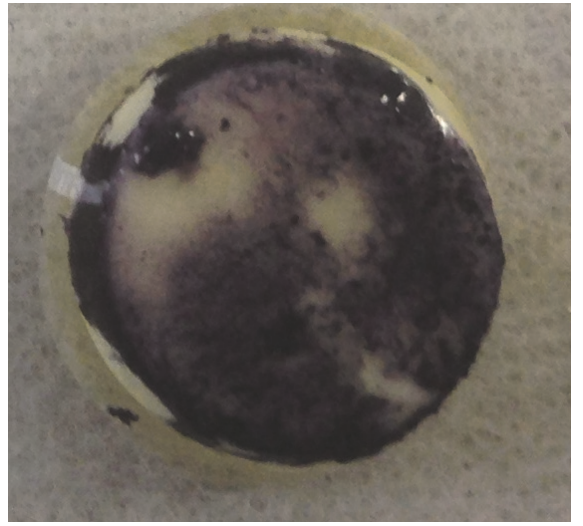

(b)

FIGURE 3: Bacterial metabolic activity in the formed biofilms. The MTT assay was used to determine the effect of SRM-S-8 on biofilm metabolic activity. Representative images are shown of (a) control biofilms formed on SRM coated HA and (b) treated biofilms formed on SRM-S-8 coated HA.

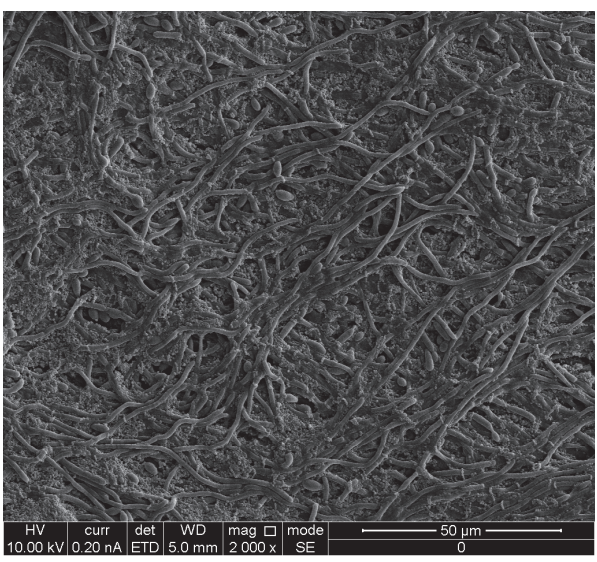

(a)

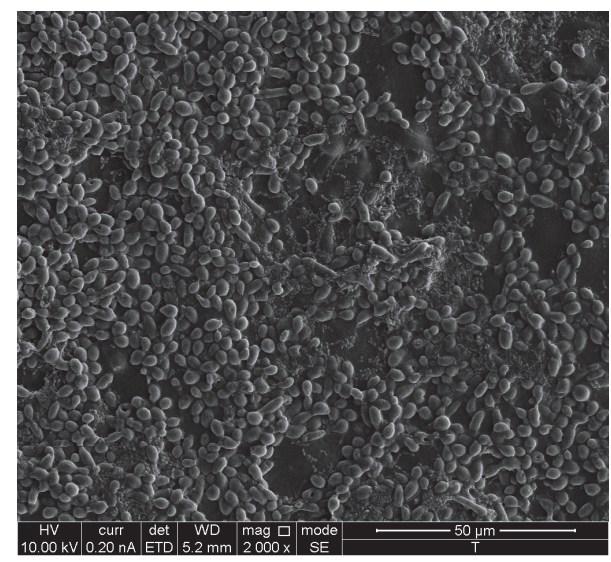

(b)

FIGURE 4: Morphology of mixed biofilm. The morphology of bacteria and fungi in the mixed biofilms formed on SRM coated HA (a) and SRM-S-8 coated HA tablets (b), visualized using Environmental High Resolution Scanning Electron Microscope at 2,000x magnification. At least three random fields were observed and analyzed, from three independent experiments.

form, which spread sporadically within the dual species biofilm. Furthermore, in spite of the firm coaggregation with candida hyphae cells in control biofilm (Figure 4(a)), only a minority of $S$. mutans cells adhered to the yeast form of C. albicans in SRM-S-8 cospecies biofilms (Figure 4(b)). Moreover, the amount of $S$. mutans cells was obviously higher in untreated controls as compared to SRM-S-8-treated samples. Furthermore, an obvious decrease was observed in the density of biofilms developed on SRM-S- 8 coated HA tablets as compared to control biofilms formed on SRM coated HA tablets.

3.4. Microbial and EPS Composition in Mixed Biofilms. CLSM analysis demonstrated an alteration of biomass and EPS production in the mixed biofilm formed onto SRMS-8 coated HA tablets as compared to control SRM coated ones. Total microbial volume and total EPS synthesis were reduced in the presence of SRM-S- 8 by $59 \%$ and $37 \%$, respectively, as compared to control (Table 1). C. albicans biomass (Figure 5(c)) and its EPS (Figure 5(d)) were reduced in the presence of SRM-S- 8 by $68 \%$ and $47 \%$, respectively, as compared to untreated control biomass and EPS (Table 1). S. mutans biomass (Figure 5(c)) and its EPS (Figure 5(d)) were decreased by $53 \%$ and $34 \%$, respectively, in the SRMS-8 treatments (Table 1) as compared to untreated control biomass. Moreover, as shown in Figures 5(c) and 5(d), total biofilm depth was reduced by $15 \mu \mathrm{m}$ (17\%) (from $90 \mu \mathrm{m}$ in control to $75 \mu \mathrm{m}$ in treated sample) in the presence of SRM-S8 coated HA tablets as compared with SRM coated HA tablets.

3.5. Profilometric Surface Analysis of Mixed Biofilms. A significant difference $(P<0.01)$ was found between biofilms formed onto SRM-S-8 coated HA tablets and control SRM coated tablets as determined by the Sq and $\mathrm{Sa}$ values (Table 2). More so the surface of control biofilms showed high roughness (Figure 6(c)) characterized by deep pits and many 


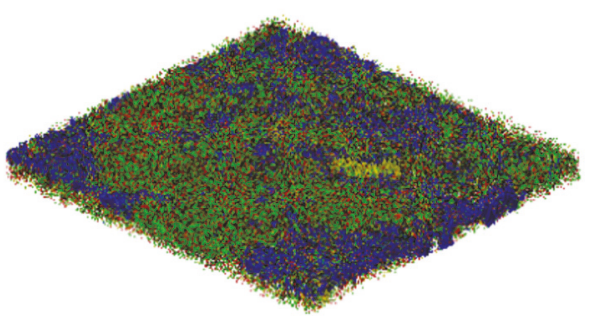

(a)

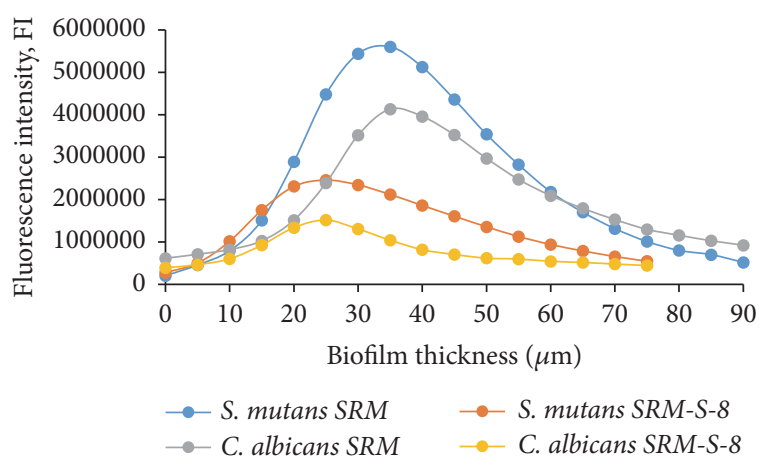

(c)

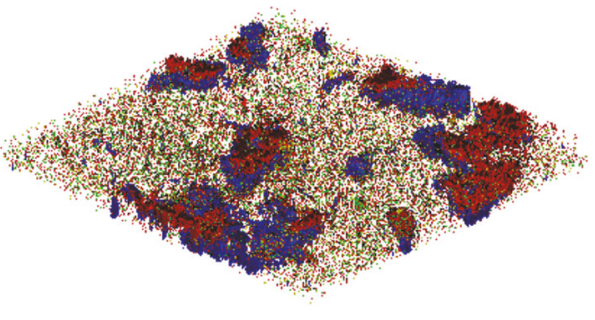

(b)

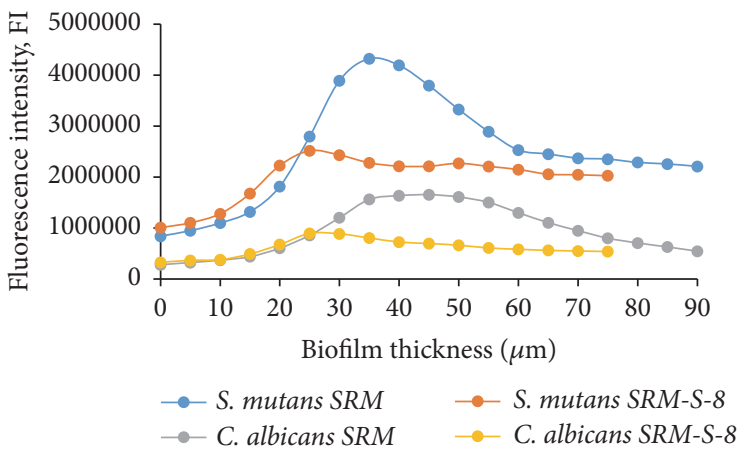

(d)

Figure 5: Microbial and EPS composition in mixed biofilms. CLSM merged images of biofilms of C. albicans and S. mutans microbial and EPS composition in control SRM coated HA (a) and treated SRM-S-8 coated HA tablets (b). Green color represents fungi and blue bacteria, yellow represents fungal EPS, and red represents bacteria. Magnification: 10x. At least three random fields were observed and analyzed, from three independent experiments. (c) Quantitative measurement of microbial composition. (d) Quantitative measurement of EPS production.

TABLE 1: The percentage of the decrease of microbes and EPS in mixed biofilm composition in the presence of SRM-S-8 as compared to control SRM (100\%).

\begin{tabular}{lc}
\hline Mixed biofilm composition & Percentage of decrease \\
\hline Total EPS & $37+/-3.48^{*}$ \\
Total microbes & $59+/-2.75^{*}$ \\
C. albicans & $68+/-4.1^{*}$ \\
S. mutans & $53+/-1.9^{*}$ \\
EPS of $C$. albicans & $47+/-2.7^{*}$ \\
EPS of $S$. mutans & $34+/-5.7^{*}$ \\
\hline
\end{tabular}

Note. ${ }^{*}$ Significantly lower than the value for the untreated control $P<0.01$.

grooves (Figure 6(a)). In contrast, biofilms treated with SRMS-8 were of smoother surface (Figure $6(d)$ ), with small pits and few grooves (Figure 6(b)).

\section{Discussion}

Since both microorganisms, C. albicans and S. mutans, exhibit virulence properties, it is of importance to control their presence in the oral cavity [40].

Our previous study demonstrated that, in its soluble state, S-8 reduced the total biomass of a cospecies C. albicansS. mutans biofilm developed in a batch environment [30]. However, the bacteria/fungi ratio was modified in favor of streptococci with increasing concentrations of soluble S-8.
TABLE 2: Surface roughness parameters of mixed biofilms formed on SRM coated HA or SRM-S-8 coated HA tablets.

\begin{tabular}{lcccc}
\hline \multirow{2}{*}{ Sample } & \multicolumn{2}{c}{$\mathrm{Sq}, \mu \mathrm{m}$} & \multicolumn{2}{c}{$\mathrm{Sa}, \mu \mathrm{m}$} \\
& Mean & SD & Mean & SD \\
\hline SRM & 11.133 & 0.170969 & 9.037 & 0.965485 \\
SRM-S-8 & 7.862 & $0.507185^{*}$ & 5.798 & $0.866531^{*}$ \\
\hline
\end{tabular}

Note. $\mathrm{SD}=$ standard deviation. ${ }^{*}$ Significantly lower than the value for the untreated control $P<0.01$.

In this study we further examined the effect of S-8 now imbedded in a SRM coating HA surface and in a continuous flow system. In addition to the reduction of total biomass by SRM-S-8, each individual microbe biofilm formation was inhibited by this pharmaceutical formulation. Previously we found that $S$. mutans are less susceptible to the soluble form of S-8 than C. albicans [30]. We proposed that sustained release of S- 8 allows better penetration of the active agent to the deeper layers of the mixed biofilm, thereby enhancing its activity against both pathogens. Indeed, it was documented that S-8 incorporated into the polymeric membrane is still active after $72 \mathrm{~h}$ of release. Furthermore, as previously reported [28], gradual release of S-8 from the SRM leads to a cumulative inhibitory effect and supports the prolonged activity of the formulation.

Our previous study [28] demonstrated the increased pharmaceutical potential of slow released S-8 against $C$. albicans monospecies biofilm. The virulence and structural characteristics of the C. albicans biofilm were affected by the 


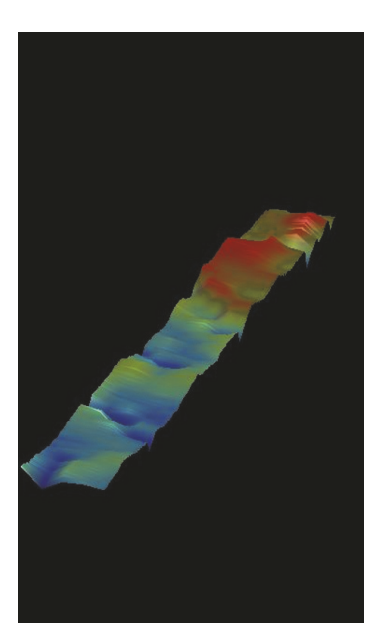

(a)

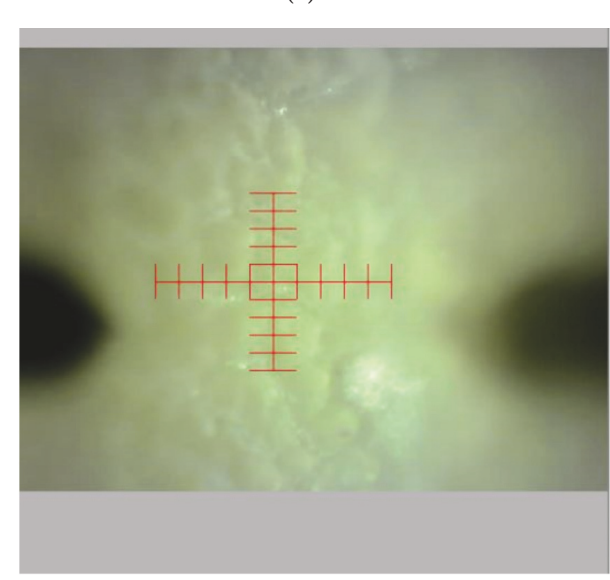

(c)

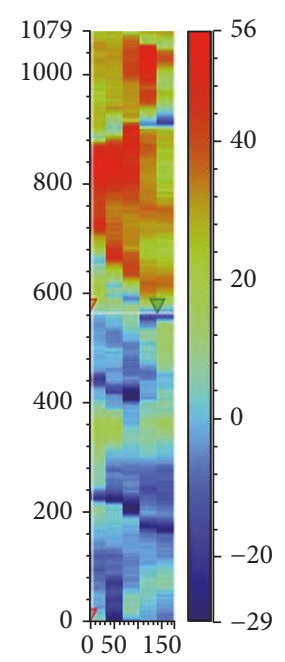

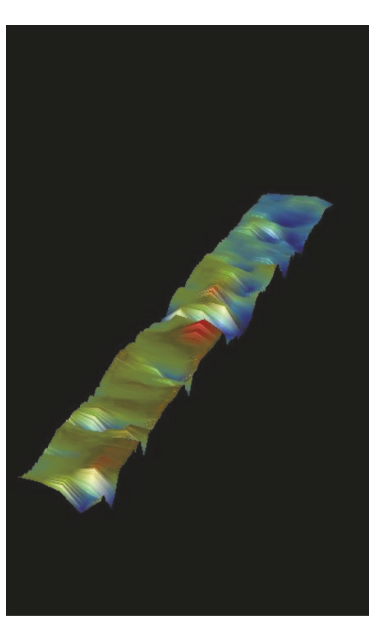

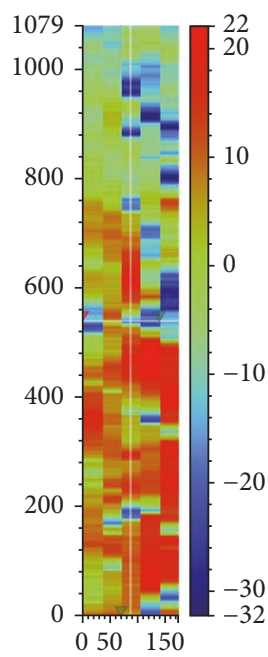

(b)

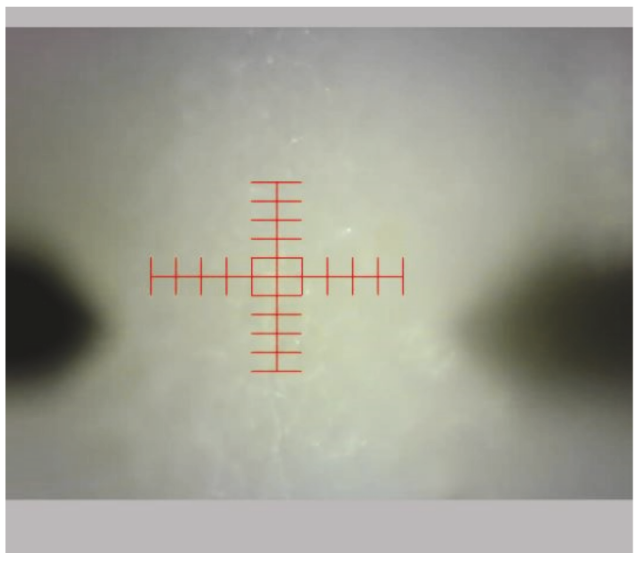

(d)

FIGURE 6: Profilometric surface analysis of mixed biofilms. 3D image of the biofilm surface topography of control SRM coated HA (a) and treated SRM-S-8 coated HA tablets (b). Biofilm images of control SRM coated HA (c) and treated SRM-S-8 coated HA (d). 10x magnification.

sustained release of S-8 [28] in a batch experimental model. In the present study, we showed that essential parameters of cospecies biofilm maintenance and maturation, such as metabolic activity, biofilm thickness, roughness, EPS production, and morphology of both pathogens, were altered by SRM-S-8 in a flow system.

The ability of $C$. albicans and $S$. mutans to maintain their growth and metabolic activity when they are together may have clinical implications for the progression of infectious diseases. Numerous agents were shown to exhibit therapeutic potential against polymicrobial biofilms, via reduction of their metabolic activity $[41,42]$. The decrease in metabolic activity of the mixed biofilm induced by SRM-S- 8 found in the present work may indicate the high efficiency of this formulation against polymicrobial infections.

EPS production plays an essential role in interactions between $C$. albicans and $S$. mutans in regard to the modulation and development of virulent cospecies biofilms. $C$. albicans and $S$. mutans enhance binding to each other in the presence of sucrose $[43,44]$. The CLSM results in our study showed reduction of total as well as each individual microbe's EPS produced in the mixed biofilm. This could be explained by the $S-8^{\prime}$ delivery mode which mediated a decrease in total as well as single pathogen biomass. Furthermore, SEM images of biofilms formed on SRM-S-8 coated HA tablets showed a decrease of either bacterial or bacteria-fungi coaggregates indicating an alteration of EPS production. Lack of EPS limits the development of a mature biofilm and results in a thinner biofilm [45]. In accordance with these findings, we demonstrated that, with treatment with the SRM-S-8, biofilms were of thinner depth due to the EPS reduction.

The surface roughness of biofilms has been demonstrated to increase bacterial attachment to biofilms in continuous flow systems [46]. It was reported that biofilms which have a nonuniform, discontinuous, and greatly heterogeneous structure are characterized by high surface roughness [45]. In our study, untreated biofilm surface appeared as highly irregular and was characterized by deep pits and numerous grooves. This structural irregularity could be attributed to the presence of long candida filaments. In contrast, biofilms 
formed on SRM-S-8 coated HA tablets demonstrated more flat and smooth surfaces due to the presence of smaller yeast forms of C. albicans.

Dissolution studies performed under conditions of continuous flow have additional value when investigating delivery systems intended for the oral cavity. We have demonstrated [27] that under static experimental conditions the release of S-8 from SRM occurred via diffusion from a homogeneous matrix with a component of erosion. In the flow-through system, the small volume of liquid, to which the coated tablet is exposed at each time point, can cause momentary saturation conditions, which leads to a slower release than in a static dissolution system under sink conditions. Therefore, testing the release in the current tested flow system provides additional valuable information for future human applications of this device.

Those results, integrated with our previous study [27], demonstrated the ability to modify the SRM formulation in order to control the release rate. The present study indicates that a single application can affect the levels of the biofilm for at least $48 \mathrm{~h}$. Future studies will be aimed at evaluating even longer-term effect of SRM-S-8 in flow-through conditions.

In conclusion, locally applied sustained-release drug delivery system can affect the dental polymicrobial biofilm, ultimately resulting in clinical improvements and a better patient compliance.

\section{Conflicts of Interest}

The authors declare that they have no conflicts of interest regarding the publication of this paper.

\section{Authors' Contributions}

Mark Feldman and Julia Shenderovich contributed equally to the manuscript.

\section{Acknowledgments}

The authors thank Dr. Mark Tarshish, Dr. Vitaly Gutkin, and Dr. Anna Radko for assistance with the CLSM, ESEM, and profilometer work, respectively.

\section{References}

[1] M. Feldman, A. Al-Quntar, I. Polacheck, M. Friedman, and D. Steinberg, "Therapeutic potential of thiazolidinedione-8 as an antibiofilm agent against Candida albicans," PLoS ONE, vol. 9, no. 5, Article ID e93225, 2014.

[2] V. K. Kutsch and D. A. Young, "New directions in the etiology of dental caries disease," Journal of the California Dental Association, vol. 39, no. 10, pp. 716-721, 2011.

[3] W. H. Bowen and H. Koo, "Biology of streptococcus mutansderived glucosyltransferases: role in extracellular matrix formation of cariogenic biofilms," Caries Research, vol. 45, no. 1, pp. 69-86, 2011.

[4] H. Koo, M. F. Hayacibara, B. D. Schobel et al., "Inhibition of Streptococcus mutans biofilm accumulation and polysaccharide production by apigenin and tt-farnesol," Journal of Antimicrobial Chemotherapy, vol. 52, no. 5, pp. 782-789, 2003.

[5] K. M. Schilling and W. H. Bowen, "Glucans synthesized in situ in experimental salivary pellicle function as specific binding sites for Streptococcus mutans," Infection and Immunity, vol. 60, no. 1, pp. 284-295, 1992.

[6] M. S. Campos, L. Marchini, L. A. S. Bernardes, L. C. Paulino, and F. G. Nobrega, "Biofilm microbial communities of denture stomatitis," Oral Microbiology and Immunology, vol. 23, no. 5, pp. 419-424, 2008.

[7] M. L. Falsetta, M. I. Klein, P. M. Colonne et al., "Symbiotic relationship between Streptococcus mutans and Candida albicans synergizes virulence of plaque biofilms in vivo," Infection and Immunity, vol. 82, no. 5, pp. 1968-1981, 2014.

[8] R. Vílchez, A. Lemme, B. Ballhausen et al., "Streptococcus mutans inhibits Candida albicans hyphal formation by the fatty acid signaling molecule trans-2-decenoic acid (SDSF)," ChemBioChem, vol. 11, no. 11, pp. 1552-1562, 2010.

[9] C. V. Bamford, A. D’Mello, A. H. Nobbs, L. C. Dutton, M. M. Vickerman, and H. F. Jenkinson, "Streptococcus gordonii modulates Candida albicans biofilm formation through intergeneric communication," Infection and Immunity, vol. 77, no. 9, pp. 3696-3704, 2009.

[10] P. I. Diaz, Z. Xie, T. Sobue et al., "Synergistic interaction between candida albicans and commensal oral streptococci in a novel in vitro mucosal model," Infection and Immunity, vol. 80, no. 2, pp. 620-632, 2012

[11] H. Xu, H. F. Jenkinson, and A. Dongari-Bagtzoglou, "Innocent until proven guilty: Mechanisms and roles of StreptococcusCandida interactions in oral health and disease," Molecular Oral Microbiology, vol. 29, no. 3, pp. 99-116, 2014.

[12] L. C. Dutton, K. H. Paszkiewicz, R. J. Silverman et al., "Transcriptional landscape of trans-kingdom communication between Candida albicans and Streptococcus gordonii," Molecular Oral Microbiology, vol. 31, no. 2, pp. 136-161, 2016.

[13] M. M. Bertolini, H. Xu, T. Sobue, C. J. Nobile, A. A. Del Bel Cury, and A. Dongari-Bagtzoglou, "Candida-streptococcal mucosal biofilms display distinct structural and virulence characteristics depending on growth conditions and hyphal morphotypes," Molecular Oral Microbiology, vol. 30, no. 4, pp. 307-322, 2015.

[14] H. Xu, T. Sobue, A. Thompson et al., "Streptococcal co-infection augments candida pathogenicity by amplifying the mucosal inflammatory response," Cellular Microbiology, vol. 16, no. 2, pp. 214-231, 2014.

[15] H. Koo and W. H. Bowen, "Candida albicans and Streptococcus mutans: a potential synergistic alliance to cause virulent tooth decay in children," Future Microbiology, vol. 9, no. 12, pp. 12951297, 2014.

[16] D. Kim, A. Sengupta, T. H. R. Niepa et al., "Candida albicans stimulates Streptococcus mutans microcolony development via cross-kingdom biofilm-derived metabolites," Scientific Reports, vol. 7, Article ID 41332, 2017.

[17] S. Gregoire, J. Xiao, B. B. Silva et al., "Role of glucosyltransferase B in interactions of Candida albicans with Streptococcus mutans and with an experimental pellicle on hydroxyapatite surfaces," Applied and Environmental Microbiology, vol. 77, no. 18, pp. 6357-6367, 2011.

[18] G. Hwang, G. Marsh, L. Gao, R. Waugh, and H. Koo, "Binding Force Dynamics of Streptococcus mutans -glucosyltransferase B to Candida albicans," Journal of Dental Research, vol. 94, no. 9, pp. 1310-1317, 2015. 
[19] D. Steinberg, T. Tal, and M. Friedman, "Sustained-release delivery systems of triclosan for treatment of Streptococcus mutans biofilm," Journal of Biomedical Materials Research - Part B Applied Biomaterials, vol. 77, no. 2, pp. 282-286, 2006.

[20] D. G. Kanjickal and S. T. Lopina, "Modeling of drug release from polymeric delivery systems - A review," Critical Reviews in Therapeutic Drug Carrier Systems, vol. 21, no. 5, pp. 345-386, 2004.

[21] D. Steinberg and M. Friedman, "Sustained-release drug delivery of antimicrobials in controlling of supragingival oral biofilms," Expert Opinion on Drug Delivery, vol. 14, no. 4, pp. 571-581, 2017.

[22] M. J. Martín, A. C. Calpena, F. Fernández, M. Mallandrich, P. Gálvez, and B. Clares, "Development of alginate microspheres as nystatin carriers for oral mucosa drug delivery," Carbohydrate Polymers, vol. 117, pp. 140-149, 2015.

[23] N. Beyth, M. Redlich, D. Harari, M. Friedman, and D. Steinberg, "Effect of sustained-release chlorhexidine varnish on Streptococcus mutans and Actinomyces viscosus in orthodontic patients," American Journal of Orthodontics and Dentofacial Orthopedics, vol. 123, no. 3, pp. 345-348, 2003.

[24] R. Czerninski, S. Sivan, D. Steinberg, I. Gati, L. Kagan, and M. Friedman, "A novel sustained-release clotrimazole varnish for local treatment of oral candidiasis," Clinical Oral Investigations, vol. 14, no. 1, pp. 71-78, 2010.

[25] G. Brackman, A. A. A. Al Quntar, C. D. Enk et al., "Synthesis and evaluation of thiazolidinedione and dioxazaborocane analogues as inhibitors of AI-2 quorum sensing in Vibrio harveyi," Bioorganic and Medicinal Chemistry, vol. 21, no. 3, pp. 660-667, 2013.

[26] O. Lidor, A. Al-Quntar, E. C. Pesci, and D. Steinberg, "Mechanistic analysis of a synthetic inhibitor of the Pseudomonas aeruginosa LasI quorum-sensing signal synthase," Scientific Reports, vol. 5, Article ID 16569, 2015.

[27] J. Shenderovich, M. Feldman, D. Kirmayer et al., "Local sustained-release delivery systems of the antibiofilm agent thiazolidinedione-8 for prevention of catheter-associated urinary tract infections," International Journal of Pharmaceutics, vol. 485, no. 1-2, pp. 164-170, 2015.

[28] M. Feldman, J. Shenderovich, A. A. A. Al-Quntar, M. Friedman, and D. Steinberg, "Sustained release of a novel anti-quorumsensing agent against oral fungal biofilms," Antimicrobial Agents and Chemotherapy, vol. 59, no. 4, pp. 2265-2272, 2015.

[29] S. Kagan, A. Jabbour, E. Sionov et al., "Anti-Candida albicans biofilm effect of novel heterocyclic compounds," Journal of Antimicrobial Chemotherapy, vol. 69, no. 2, Article ID dkt365, pp. 416-427, 2014.

[30] M. Feldman, I. Ginsburg, A. Al-Quntar, and D. Steinberg, "Thiazolidinedione-8 alters symbiotic relationship in C. albicans-S. mutans dual species biofilm," Frontiers in Microbiology, vol. 7, article no. 140, 2016.

[31] T. D. Morgan and M. Wilson, "The effects of surface roughness and type of denture acrylic on biofilm formation by Streptococcus oralis in a constant depth film fermentor," Journal of Applied Microbiology, vol. 91, no. 1, pp. 47-53, 2001.

[32] J. Pratten, "Growing oral biofilms in a constant depth film fermentor (CDFF).," Current protocols in microbiology, vol. 1, p. 1B.5, 2007.

[33] D. Steinberg, M. Feldman, I. Ofek, and E. I. Weiss, "Cranberry high molecular weight constituents promote Streptococcus sobrinus desorption from artificial biofilm," International Journal of Antimicrobial Agents, vol. 25, no. 3, pp. 247-251, 2005.
[34] C. Lüdecke, K. D. Jandt, D. Siegismund et al., "Reproducible biofilm cultivation of chemostat-grown escherichia coli and investigation of bacterial adhesion on biomaterials using a nonconstant-depth film fermenter," PLoS ONE, vol. 9, no. 1, Article ID e84837, 2014.

[35] D. M. Deng and J. M. Ten Cate, "Demineralization of dentin by Streptococcus mutans biofilms grown in the constant depth film fermentor," Caries Research, vol. 38, no. 1, pp. 54-61, 2004.

[36] M. Gerami-Nejad, L. F. Zacchi, M. McClellan, K. Matter, and J. Berman, "Shuttle vectors for facile gap repair cloning and integration into a neutral locus in Candida albicans," Microbiology (United Kingdom), vol. 159, no. 3, pp. 565-579, 2013.

[37] D. Assaf, D. Steinberg, and M. Shemesh, "Lactose triggers biofilm formation by Streptococcus mutans," International Dairy Journal, vol. 42, pp. 51-57, 2015.

[38] J. Chandra, D. M. Kuhn, P. K. Mukherjee, L. L. Hoyer, T. McCormick, and M. A. Ghannoum, "Biofilm formation by the fungal pathogen Candida albicans: development, architecture, and drug resistance," Journal of Bacteriology, vol. 183, no. 18, pp. 5385-5394, 2001.

[39] J. F. Lasserre, S. Toma, T. Bourgeois, H. El Khatmaoui, E. Marichal, and M. C. Brecx, "Influence of low direct electric currents and chlorhexidine upon human dental biofilms," Clinical and Experimental Dental Research, vol. 2, no. 2, pp. 146-154, 2016.

[40] C. C. Sheth, K. Makda, Z. Dilmahomed et al., "Alcohol and tobacco consumption affect the oral carriage of Candida albicans and mutans streptococci," Letters in Applied Microbiology, vol. 63, no. 4, pp. 254-259, 2016.

[41] B. M. Peters, R. M. Ward, H. S. Rane, S. A. Lee, and M. C. Noverr, "Efficacy of ethanol against Candida albicans and Staphylococcus aureus polymicrobial biofilms," Antimicrobial Agents and Chemotherapy, vol. 57, no. 1, pp. 74-82, 2013.

[42] S. Dashper, N. O’Brien-Simpson, S. W. Liu et al., "Oxantel disrupts polymicrobial biofilm development of periodontal pathogens," Antimicrobial Agents and Chemotherapy, vol. 58, no. 1, pp. 378-385, 2014.

[43] C. Branting, M.-L. Sund, and L. E. Linder, "The influence of Streptococcus mutans on adhesion of Candida albicans to acrylic surfaces in vitro," Archives of Oral Biology, vol. 34, no. 5, pp. 347-353, 1989.

[44] H. F. Jenkinson, H. C. Lala, and M. G. Shepherd, "Coaggregation of Streptococcus sanguis and other streptococci with Candida albicans," Infection and Immunity, vol. 58, no. 5, pp. 1429-1436, 1990.

[45] M.-Y. Wu, V. Sendamangalam, Z. Xue, and Y. Seo, "The influence of biofilm structure and total interaction energy on Escherichia coli retention by Pseudomonas aeruginosa biofilm," Biofouling, vol. 28, no. 10, pp. 1119-1128, 2012.

[46] T. J. Battin, L. A. Kaplan, J. D. Newbold, and C. M. E. Hansen, "Contributions of microbial biofilms to ecosystem processes in stream mesocosms," Nature, vol. 426, no. 6965, pp. 439-442, 2003. 

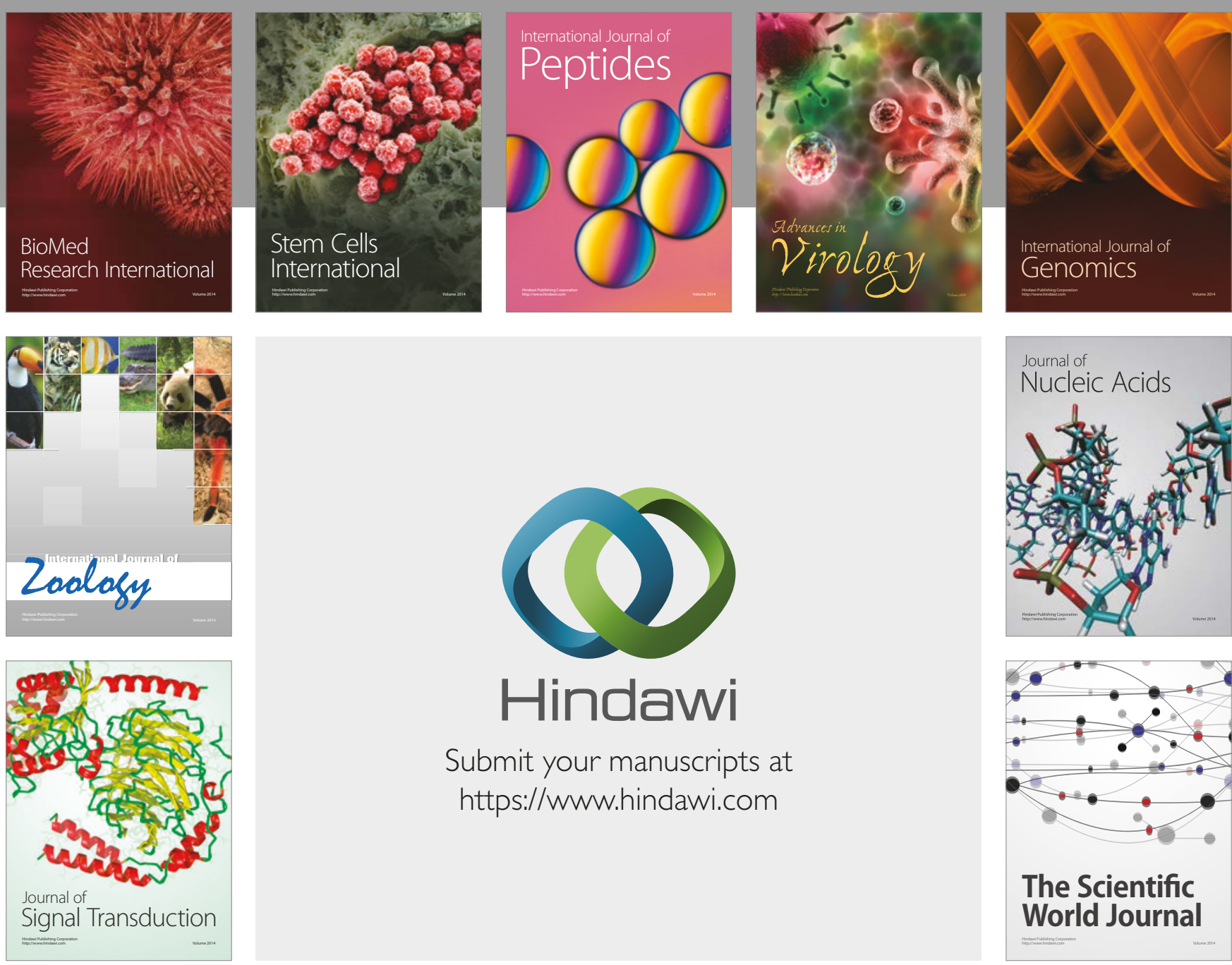

Submit your manuscripts at

https://www.hindawi.com
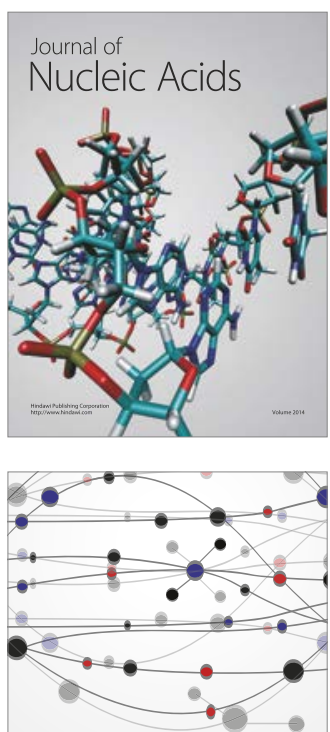

The Scientific World Journal

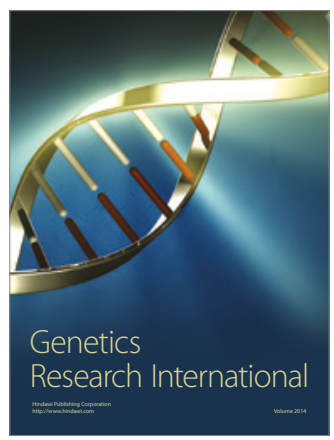

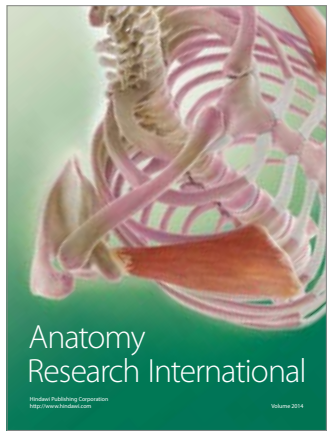

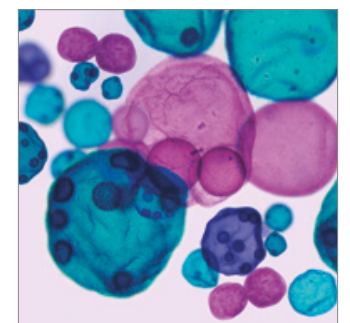

International Journal of Microbiology
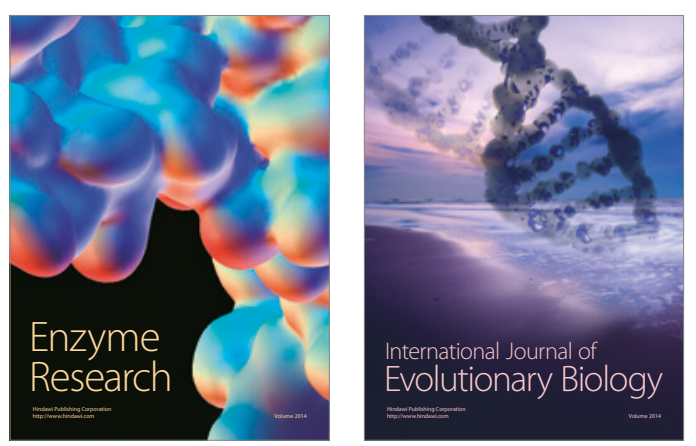
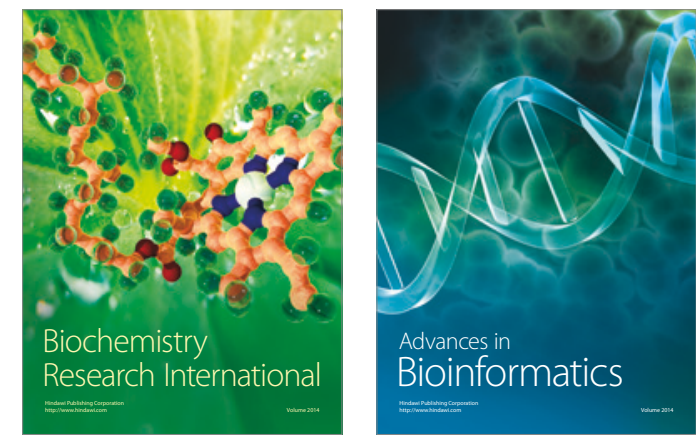

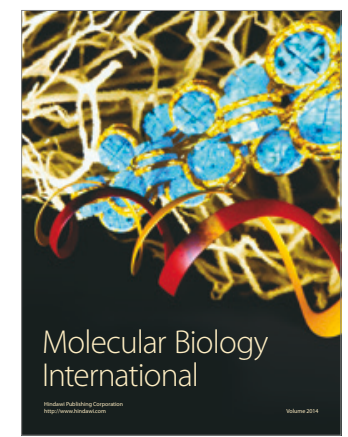

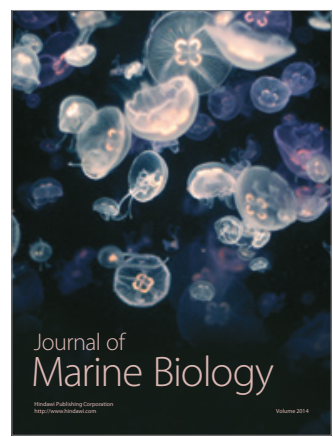

